\title{
Aferição do volume residual gástrico: retrato da prática clínica de enfermeiros
}

\author{
Assessing gastric residual volume: a description of nurses' clinical practice \\ Verificación del volumen gástrico residual: retrato de la práctica clínica de enfermeros
}

Vanessa de Brito Poveda ${ }^{1}$, Ana Carolina Breviglieri Alves Castilho ${ }^{2}$, Lilia de Souza Nogueira ${ }^{1}$, Renata Eloah Lucena

Ferretti-Rebustini ${ }^{1}$, Rita de Cássia Gengo e Silva ${ }^{1}$

Como citar este artigo:

Poveda VB, Castilho ACBA, Nogueira LS, Ferretti-Rebustini REL, Silva RCG. Assessing gastric residual volume: a description of nurses' clinical practice. Rev Esc Enferm USP. 2018;52:e03352. DOI: http://dx.doi.org/10.1590/S1980-220X2017038803352

${ }^{1}$ Universidade de São Paulo, Escola de Enfermagem, Departamento de Enfermagem Médico Cirúrgica, São Paulo, SP, Brasil.

${ }^{2}$ Universidade de São Paulo, Escola de Enfermagem, São Paulo, SP, Brasil.

\begin{abstract}
Objective: To analyze the differences in nurses' clinical practice for assessing residual gastric volume and identifying the theoretical framework which supports their practice. Method: A cross-sectional study carried out by sending an online questionnaire by e-mail to nurses registered at the Regional Nursing Council of the State of São Paulo. Results: This study included 598 nursing professionals, with 484 only providing care to adults and 114 exclusively to children. The gastric residual volume test is performed by $83.4 \%$ of nursing professionals; in most cases the suspension and prescription of enteral nutritional therapy are performed by the physician. Suspension of enteral nutritional therapy among adults predominantly occurs when the gastric residual volume is equal to $200 \mathrm{ml}$, and in children when values are less than $100 \mathrm{ml}$. Procedure after diet suspension involves the return of aspirated gastric contents and maintaining the catheter closed until the next hour in $48.3 \%$ of the procedures among adults, and $68.4 \%$ among children. $42.9 \%$ of the participants in this study were not aware of the theoretical basis that supports the test performance. Conclusion: We can highlight the need for nurses' training and further studies focused on the practice for assessing gastric residual volume.
\end{abstract}

DESCRIPTORS

Diet; Enteral Nutrition; Gastric Emptying; Nursing Care. 


\section{INTRODUÇÃO}

A terapia nutricional enteral (TNE) tem sofrido um crescimento importante ao longo dos últimos anos, aliado ao desenvolvimento científico que a subsidia, e, os benefícios associados ao início precoce da terapia nutricional entre pacientes graves com problemas nutricionais refletem em melhora da função imune e diminuição de complicações metabólicas, melhorando o prognóstico dos pacientes ${ }^{(1)}$.

Dentro deste contexto, a equipe de enfermagem tem papel de destaque na equipe multidisciplinar, atuando na identificação, suporte, administração e evolução clínica dos pacientes em terapia nutricional. Portanto, esses profissionais se deparam frequentemente com os problemas associados à administração da TNE, dentre eles, o volume residual gástrico (VRG), ou seja, a não adequada absorção do volume da TNE administrado, que pode ser um indicativo de intolerância gástrica e impactar diretamente na continuidade da administração da terapia ${ }^{(2)}$.

A prática da análise do VRG é frequente nas unidades de cuidados intensivos; contudo, constitui ainda uma prática controversa. Atualmente, diversas entidades internacionais, como a American Society for Parenteral and Enteral Nutrition (ASPEN), e nacionais, como a Sociedade Brasileira de Nutrição Enteral e Parenteral (SBNPE) e a Associação Brasileira de Nutrologia (ABRAN), ressaltam a carência de estudos que subsidiem a forma mais adequada de se analisar o VRG e se este está ou não associado a melhor prognóstico ou ocorrência de complicações, como aumento da incidência de pneumonia por regurgitação e aspiração, além de haver dificuldade em estabelecerem-se padrões a serem seguidos em relação ao volume ${ }^{(3-4)}$.

Nas orientações nacionais recomenda-se a mensuração do VRG conforme protocolo institucional, embora não se estabeleçam critérios a serem seguidos, deixando em aberto a questão sobre como avaliar os valores encontrados e, frente a eles, quais medidas devem ser implementadas ${ }^{(3)}$.

Internacionalmente, a ASPEN recomenda que VRG entre 200 e $500 \mathrm{ml}$ merecem atenção por parte da equipe multidisciplinar, recomendando a implementação de medidas que reduzam o risco de aspiração; no entanto, esta entidade não recomenda a suspensão da dieta em valores de VRG inferiores a $500 \mathrm{ml}$, na ausência de outros sinais indicativos de intolerância ${ }^{(4)}$.

Dessa forma, considerando que as recomendações internacionais vigentes geram dúvidas e as recomendações nacionais estimulam que os hospitais criem protocolos próprios, este estudo teve como objetivo conhecer a prática de manejo do volume residual gástrico (VRG) entre enfermeiros clínicos de diferentes unidades de saúde do estado de São Paulo e identificar a fundamentação teórica que subsidia a prática.

\section{MÉTODO}

Trata-se de estudo transversal realizado por meio do envio de um questionário online aos e-mails dos enfermeiros cadastrados no Conselho Regional de Enfermagem do Estado de São Paulo (COREn-SP).

O questionário proposto pelos autores incluía questões relacionadas à caracterização sócio demográfica dos participantes e aspectos relacionados à prática, ou não, da avaliação do VRG pelos enfermeiros em diferentes unidades de saúde. Sua construção foi fundamentada em aspectos apontados pela American Society for Parenteral and Enteral Nutrition (ASPEN), Sociedade Brasileira de Nutrição Enteral e Parenteral (SBNPE) e Associação Brasileira de Nutrologia (ABRAN) ${ }^{(3-4)}$.

O link do questionário proposto pelos autores passou por validação aparente e de conteúdo por três enfermeiros com título de doutor e experiência no manuseio de cateteres nasogástricos e/ou entéricos e utilização da medida de VRG em suas práticas clínicas. Os juízes avaliaram a qualidade de apresentação das questões e a completude das informações. Os juízes sugeriram alterações, no sentido de tornar as questões e alternativas mais claras, que foram acatadas pelos autores.

A coleta de dados ocorreu por meio do envio dos instrumentos aos enfermeiros que possuam conta de e-mail, cadastrados no COREn-SP, até outubro de 2014. O instrumento de coleta de dados foi hospedado em um site específico para esta finalidade. Este site possui um sistema incluído ao questionário, que não permite ao enfermeiro progredir no preenchimento das informações, caso não seja assinalada a concordância com o TCLE e também não permitia pular questões essenciais à avaliação dos quesitos objetos de estudo.

Dessa forma, o preenchimento dos questionários foi aguardado por três meses, ou seja, noventa dias, a partir do envio das cartas convite; após esta ocasião, o preenchimento do instrumento foi bloqueado pelos pesquisadores.

Dos 92.000 e-mails enviados aos enfermeiros, foram recebidas 1.014 respostas a consulta realizada até a data limite proposta. Destas, foram incluídos 598 respondentes, que concordaram em participar da investigação, assinalando esta intenção no Termo de Consentimento Livre e Esclarecido (TCLE) e que responderam completamente o instrumento enviado.

Destaca-se que apesar do envio ter sido realizado para todos os e-mails cadastrados COREn-SP, sabe-se que situações que não podiam ser controladas pelos autores, como dificuldades relacionadas aos provedores de e-mails, caixas lotadas de mensagens e e-mails desatualizados ou incorretos podem ter afetado a quantidade de respostas recebidas.

A análise dos dados foi realizada de forma descritiva. Os resultados foram apresentados segundo distribuições de frequências e medidas estatísticas descritivas tais como média aritmética, desvio-padrão, mediana, valores mínimo e máximo para as variáveis quantitativas. Foi utilizada a técnica de dupla digitação de dados e os dados coletados foram analisados por meio do software Statistical Package Social Sciences (SPSS), versão 20.0.

Este projeto recebeu aprovação do Comitê de Ética em Pesquisa da Escola de Enfermagem da Universidade de São Paulo, recebendo número do parecer 544.073/2014, atendendo as Diretrizes e Normas Regulamentadoras de Pesquisas envolvendo seres humanos, a partir da Resolução no. 466 de 2012, do Conselho Nacional de Saúde.

\section{RESULTADOS}

A amostra foi composta por 598 profissionais da enfermagem, em sua maioria do sexo feminino ( $511 ; 85,5 \%)$, dos quais $445(74,4 \%)$ referiam ser especialistas (Tabela 1$)$, com idade média de 33,10 anos ( $\mathrm{DP} \pm$ 7,6 anos), mínimo de 22 e máximo de 59 anos. 
A maioria desenvolve atividades em hospitais públicos $(261 ; 44,1 \%)$ ou privados $(245 ; 41,4 \%)$, destacando-se as Unidades de Terapia Intensiva (UTI) $(226 ; 37,8 \%)$ e de Internação Clínico-Cirúrgica (216; 36,1\%), atendendo especialmente adultos $(484 ; 80,9 \%)$ (Tabela 1$)$.

Tabela 1 - Caracterização sócio-demográfica - São Paulo, SP, Brasil, 2016.

\begin{tabular}{|c|c|c|}
\hline Variáveis & $\mathbf{N}$ & $\%$ \\
\hline \multicolumn{3}{|l|}{ Sexo } \\
\hline Masculino & 87 & 14,5 \\
\hline Feminino & 511 & 85,5 \\
\hline \multicolumn{3}{|l|}{ Nível de formação } \\
\hline Especialização & 445 & 74,4 \\
\hline Mestrado & 32 & 5,4 \\
\hline Doutorado & 8 & 1,3 \\
\hline Pós-doutorado & 7 & 1,2 \\
\hline Em branco/ Não tem o título & 106 & 17,7 \\
\hline \multicolumn{3}{|l|}{ Tipo de Instituição* } \\
\hline Hospital Público & 261 & 44,1 \\
\hline Hospital Privado & 245 & 41,4 \\
\hline Instituição de Longa Permanência & 54 & 9,1 \\
\hline Ambulatório & 17 & 2,9 \\
\hline Outros & 15 & 2,5 \\
\hline \multicolumn{3}{|l|}{ Área de Atuação } \\
\hline Unidade de Terapia Intensiva & 226 & 37,8 \\
\hline Clínica médico-cirúrgica & 216 & 36,1 \\
\hline Emergência & 55 & 9,2 \\
\hline Ambulatório & 23 & 3,8 \\
\hline Outros & 78 & 13,1 \\
\hline \multicolumn{3}{|l|}{ Público Atendido } \\
\hline Adultos & 484 & 80,9 \\
\hline Crianças & 114 & 19,1 \\
\hline
\end{tabular}

*6 Missing. Nota: $(\mathrm{N}=598)$.

Dos 598 enfermeiros da casuística, 83,4\% (n=499) realizam a prática do teste de VRG em suas unidades. Em sua maioria, a equipe de enfermagem é responsável pelo teste do VRG, com destaque ao enfermeiro $(497 ; 83,1 \%)$, seguido pelo técnico de enfermagem $(451 ; 75,4 \%)$ e o auxiliar de enfermagem (249; 41,6\%). Os dispositivos mais comumente utilizados nas unidades em que os enfermeiros atuam são os cateteres de Dobbhoff (414; 69,2\%) ou de Levine (335; $56,0 \%$ ) (Tabela 2).

A fundamentação teórica que embasa a prática do teste de VRG mais citada foi a SBNPE $(137 ; 22,9 \%)$ seguida da Comissão Multiprofissional de Alimentação Enteral e Parenteral (CMAEP) (128; 21,4\%) e da ASPEN (93; $15,5 \%)$. No entanto, destacou-se também, respostas de profissionais que relatavam em relação à fundamentação teórica seguir apenas as orientações de médicos $(77 ; 12,9 \%)$ ou de nutricionistas $(71 ; 11,9 \%)$. Destaca-se ainda que 184 $(30,8 \%)$ profissionais relataram desconhecimento quanto à fundamentação teórica da prática e $2(12,2 \%)$ realizavam a prática sem fundamentação (Tabela 2).

Tabela 2 - Distribuição das características sobre a prática do teste de volume residual gástrico - São Paulo-SP, Brasil, 2016.

\begin{tabular}{|c|c|c|}
\hline Variáveis & $\mathbf{N}$ & $\%$ \\
\hline \multicolumn{3}{|l|}{ Realização do teste de VRG ( $\mathrm{N}=598$ ) } \\
\hline Sim & 499 & 83,4 \\
\hline Não & 98 & 16,4 \\
\hline Sem resposta & 1 & 0,2 \\
\hline \multicolumn{3}{|l|}{ Profissional que realiza o teste de VRG* } \\
\hline Enfermeiro & 497 & 83,1 \\
\hline Técnico de enfermagem & 451 & 75,4 \\
\hline Auxiliar de enfermagem & 249 & 41,6 \\
\hline Nutricionista & 2 & 0,3 \\
\hline Médico & 2 & 0,3 \\
\hline \multicolumn{3}{|l|}{ Tipo de dispositivo utilizado pelos pacientes* } \\
\hline Cateter Dobbhoff & 414 & 69,2 \\
\hline Cateter Levine & 335 & 56,0 \\
\hline Gastrostomia & 217 & 36,3 \\
\hline Jejunostomia & 99 & 16,7 \\
\hline \multicolumn{3}{|l|}{ Fundamentação teórica para a prática* } \\
\hline $\begin{array}{l}\text { American Society for Parenteral and Enteral Nutrition } \\
\text { (ASPEN) }\end{array}$ & 93 & 15,6 \\
\hline $\begin{array}{l}\text { Sociedade Brasileira de Nutrição Enteral e Parenteral } \\
\text { (SBNPE) }\end{array}$ & 137 & 22,9 \\
\hline $\begin{array}{l}\text { Comissão Multiprofissional de Alimentação Enteral e } \\
\text { Parenteral (CMAEP) }\end{array}$ & 128 & 21,4 \\
\hline Orientação do médico & 77 & 12,9 \\
\hline Orientação do nutricionista & 71 & 11,9 \\
\hline Desconhece & 184 & 30,8 \\
\hline Sem fundamentação & 12 & 2,0 \\
\hline
\end{tabular}

Legenda: *Questão com possibilidade de mais de uma resposta.

Entre aqueles que prestam assistência a pacientes adultos (484; 80,9\%), em $220(45,4 \%)$ casos os enfermeiros adotam o volume de $200 \mathrm{ml}$ como indicativo de suspensão da TNE. Entre crianças, a maior parte referiu volumes menores do que $100 \mathrm{ml}(32 ; 28,1 \%)$, no entanto, nota-se valores que podem ser considerados conflitantes, uma vez que houve referência a $600 \mathrm{ml}$, em $1(0,9 \%)$ caso, como indicativo de suspensão da TNE (Tabela 3).

Independente do público atendido, os enfermeiros participantes referiram ser o médico o profissional responsável pela suspensão da dieta, em $352(72,7 \%)$ dos casos em adultos e 109 (95,6\%) das situações em crianças (Tabela 3).

Em relação à conduta realizada após a suspensão da dieta, entre aqueles que atendem adultos, $234(48,3 \%)$ devolvem o conteúdo gástrico, considerado excedente, anteriormente 
aspirado e a sonda permanece fechada até o próximo horário; 206 (42,6\%), ao detectar volume excedente, o desprezam e a dieta é instalada apenas no próximo horário; $55(11,4 \%)$ relatam administração de medicamentos e $40(8,3 \%)$ relatam outras condutas (Tabela 3).

Entre os que atendem crianças, 78 (68,4\%) devolvem o conteúdo gástrico, considerado excedente, anteriormente aspirado e a sonda permanece fechada até o próximo horário; $26(22,8 \%)$ desprezam o volume excedente e instalam a dieta no próximo horário; $19(16,7 \%)$ relatam outras condutas e três $(2,6 \%)$ a administração de medicamentos (Tabela 3).

Independente do atendimento de crianças ou de adultos, os profissionais responsáveis para a liberação da TNE são similares, sendo para adultos o médico $(368 ; 76,0 \%)$, o enfermeiro (198; 40,9\%) e o nutricionista $(143 ; 29,5 \%)$. Entre crianças destacam-se o médico (105; 92,1\%), seguido pelo enfermeiro $(23 ; 20,2 \%)$ e, por fim, pelo nutricionista $(12 ; 10,5 \%)$ (Tabela 3$)$.

Tabela 3 - Descrição sobre liberação e suspensão da dieta após teste de volume residual gástrico - São Paulo, SP, Brasil, 2016.

\begin{tabular}{|c|c|c|c|c|}
\hline \multirow{3}{*}{ Variáveis } & \multicolumn{4}{|c|}{ Clientela atendida } \\
\hline & \multicolumn{2}{|c|}{ Adultos } & \multicolumn{2}{|c|}{ Crianças } \\
\hline & $\mathbf{N}$ & $\%$ & $\mathbf{N}$ & $\%$ \\
\hline \multicolumn{5}{|l|}{ Volume indicado para suspensão da dieta* } \\
\hline Menos de $100 \mathrm{ml}$ & 99 & 20,4 & 32 & 28,1 \\
\hline $200 \mathrm{ml}$ & 220 & 45,4 & 13 & 11,4 \\
\hline $300 \mathrm{ml}$ & 53 & 11,0 & 3 & 2,6 \\
\hline $400 \mathrm{ml}$ & 17 & 3,5 & - & - \\
\hline $500 \mathrm{ml}$ & 35 & 7,2 & 2 & 1,7 \\
\hline $600 \mathrm{ml}$ & 8 & 1,7 & 1 & 0,9 \\
\hline Outros & 44 & 9,1 & 62 & 54,4 \\
\hline Não respondeu & 8 & 1,7 & 1 & 0,9 \\
\hline \multicolumn{5}{|l|}{ Profissional responsável pela suspensão da dieta* } \\
\hline Médico & 352 & 72,7 & 109 & 95,6 \\
\hline Enfermeiro & 262 & 54,1 & 37 & 32,5 \\
\hline Nutricionista & 138 & 28,5 & 8 & 7,0 \\
\hline Técnico de enfermagem & 5 & 1,0 & 0 & 0 \\
\hline Auxiliar de enfermagem & 3 & 0,6 & 0 & 0 \\
\hline \multicolumn{5}{|l|}{ Conduta após suspender a dieta* } \\
\hline Devolver o conteúdo aspirado ao paciente e com sonda fechada até o próximo horário & 234 & 48,3 & 78 & 68,4 \\
\hline Desprezar volume excedente e instalar dieta no próximo horário & 206 & 42,6 & 26 & 22,8 \\
\hline Uso de medicamentos & 55 & 11,4 & 3 & 2,6 \\
\hline Outros & 40 & 8,3 & 19 & 16,7 \\
\hline \multicolumn{5}{|l|}{ Profissional responsável pela liberação da dieta* } \\
\hline Médico & 368 & 76,0 & 105 & 92,1 \\
\hline Enfermeiro & 198 & 40,9 & 23 & 20,2 \\
\hline Nutricionista & 143 & 29,5 & 12 & 10,5 \\
\hline
\end{tabular}

* Questão com possibilidade de mais de uma resposta.

\section{DISCUSSÃO}

Em sua maioria, os profissionais de enfermagem participantes desta investigação e que realizam o teste de VRG possuem especialização em sua formação e atuam em UTI ou em unidades de internação Clínico-Cirúrgica, uma vez que a desnutrição e outras indicações para a TNE, como o suprimento alimentar insuficiente, acidentes cerebrovasculares, impedimentos motores, trauma, entre outras, aliado à complexidade dos pacientes atendidos, estão mais presentes nestas unidades ${ }^{(5-7)}$.

A TNE tem como finalidade a manutenção e recuperação do estado nutricional por meio da ingesta controlada de nutrientes para substituir ou completar a alimentação em pacientes impossibilitados de ingestão oral, como os acometidos por patologias do trato gastrointestinal, intubação orotraqueal ou distúrbios neurológicos com comprometimento do nível de consciência, sendo indicada para pacientes incapacitados de alcançar pelo menos $70 \%$ de suas recomendações nutricionais diárias ${ }^{(4,8)}$. Contraindica-se a TNE em situações em que o trato gastrointestinal não está íntegro e/ou funcional, como em situações de íleo paralítico, obstrução mecânica do trato gastrointestinal (TGI), vômitos e diarreia severa ${ }^{(9)}$.

Os dispositivos para a TNE mais presentes no estudo foram o cateter Dobbhoff, em geral empregado em posição pós-pilórica e indicado para pacientes com risco de aspiração, gastroparesia e retardo do esvaziamento gástrico ${ }^{(7)}$. Já o cateter Levine, recomendado para alocamento gástrico, permite maior tolerância a uma variedade de fórmulas e maior volume de dieta infundida, o que pode ocasionar maior risco de aspiração; por outro lado, destaca-se o melhor custo-efetividade deste cateter quando comparado às gastrostomias ou jejunostomias ${ }^{(10)}$. 
Analisando estes aspectos, metanálise recente observou que a posição pós-pilórica pode resultar em melhor oferta nutricional, além de diminuir o VRG; no entanto, não está claro se os resultados clínicos associados à aspiração, pneumonia ou mortalidade apresentam melhora com este posicionamento do cateter ${ }^{(11)}$.

A intolerância à TNE é frequentemente usada como sinônimo de disfunção do TGI, acomete até $50 \%$ dos pacientes submetidos à ventilação mecânica e se caracteriza por lento esvaziamento gástrico, levando a sinais e sintomas associados ao VRG elevado, com potencial risco de refluxo e de aspiração, somado a presença de alterações gastrointestinais como distensão abdominal, constipação, vômitos, diarreia, aumento da circunferência abdominal e desconforto subjetivo ${ }^{(2,4)}$.

Apesar da intolerância a TNE estar associada a diversos aspectos, é frequente observar que a equipe de enfermagem utiliza a medição do VRG como a única forma de aferição da alteração, fato observado em estudo longitudinal americano avaliando 332 enfermeiros, que identificou que $89 \%$ dos entrevistados utilizam esta estratégia na avaliação da intolerância alimentar ${ }^{(12)}$. Neste mesmo sentido, investigação pregressa verificou que entre 2298 respondentes, $97,1 \%$ dos enfermeiros utilizam a avaliação do VRG como sinal de intolerância a TNE, apesar de outros aspectos também terem sido citados, tais como avaliação da distensão abdominal $(88,5 \%)$, vômitos $(86,0 \%)$, ausculta de sons abdominais $(79,7 \%)$, náusea $(79,6 \%)$ e desconforto gástrico $(79,3 \%)^{(13)}$.

Este aspecto também foi evidenciado no presente estudo ao observar que a prática do teste de VRG foi relatada pela maioria da amostra, sendo que, entre aqueles profissionais que atendem adultos, grande parte suspende a dieta quando há presença de VRG igual ou superior a $200 \mathrm{ml} \mathrm{e}$, entre crianças, valores inferiores a $100 \mathrm{ml}$, sendo que a conduta instituída envolve, predominantemente, a devolução do conteúdo gástrico excedente aspirado e o fechamento do cateter até o próximo horário de administração da TNE.

Observa-se na literatura controvérsias em relação à padronização relativa à melhor forma de atuação frente ao $V_{R G}^{(2)}$ entre os guias nacionais e internacionais. Neste sentido, a recomendação da ASPEN sugere avaliar o VRG a cada seis horas ou antes de cada infusão, quando em uso da alimentação intermitente. Caso o VRG seja superior a $500 \mathrm{ml}$, deve-se interromper a dieta por duas horas e reavaliar o VRG, reiniciando a dieta se o volume aspirado for inferior a $500 \mathrm{ml}$ e interrompendo se houver manutenção do volume ${ }^{(14)}$.

No Brasil, a SBNPE e a ABRAN afirmam não haver consenso quanto aos valores ideais ${ }^{(3)}$. No entanto, destaca-se que as orientações nacionais não apresentam atualizações desde 2011.

Resultados de VRG elevados são considerados parâmetros indicativos de desordens associadas ao esvaziamento gástrico, pneumonia, regurgitação e aspiração e que geram a cessação da administração da TNE, mais frequentemente quando são encontrados valores entre $200 \mathrm{ml} \mathrm{e} 250 \mathrm{ml}^{(2,4,13)}$. Embora estudo tenha detectado que pelo menos um terço dos enfermeiros relata a interrupção da TNE se VRG igual ou menor a $150 \mathrm{ml}^{(13)}$.
Destaca-se que, o VRG, como marcador para risco de aspiração, não deve ser utilizado de forma padronizada entre pacientes críticos. Para aqueles com VRG entre 200 e $500 \mathrm{ml}$, sugere-se a implementação de medidas de redução do risco de aspiração, evitando a suspensão da TNE apenas baseada em avaliação de VRG, sem outros sintomas de intolerância alimentar; no entanto, ressalta-se que a qualidade de evidência que suporta esta recomendação é considerada baixa ${ }^{(4)}$.

Estudo objetivando avaliar a associação de intolerância a TNE e mortalidade em 1712 pacientes utilizando a terapia em UTI, observou que os mais frequentes sintomas foram vômitos, ausência de sons abdominais, distensão abdominal

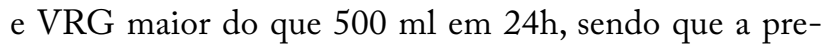
sença de três ou cinco destes sintomas foram associados à maior mortalidade ${ }^{(15)}$.

Dentre os que atendem crianças, dada à diversidade de faixa etária, encontram-se sugestões diversas na literatura científica quanto aos valores a serem considerados, desde estudo que aponta como elevado um VRG maior que 10 $\mathrm{ml} / \mathrm{kg}$ para crianças e volumes acima de $400 \mathrm{ml}$ para adolescentes de até $50 \mathrm{~kg}$, sendo recomendado, nestes casos, a interrupção da TNE e reavaliação do VRG com alteração do volume de infusão da dieta, caso necessário, e a instituição de agente pró-cinético ${ }^{(16)}$, até pesquisadores que sugerem uma abordagem percentual na avaliação do VRG em crianças, definindo intolerância a TNE quando o VRG é maior que $50 \%$ da dieta infundida ou na presença de vômitos e distensão abdominal ${ }^{(17)}$.

Neste estudo, observou-se que a maior parte dos enfermeiros participantes citaram os médicos como os profissionais responsáveis pela suspensão e liberação da TNE, o que vem de encontro a legislação nacional, que estabelece que o médico é o profissional responsável pela indicação e prescrição médica da TNE e o nutricionista pela prescrição dietética da TNE, ou seja, do tipo e a quantidade dos nutrientes adequados ao paciente, considerando a patologia e as condições do trato digestivo. Já ao enfermeiro cabem diversas atividades relacionadas, principalmente, a administração da TNE, envolvendo também a educação dos pacientes e familiares ${ }^{(18)}$.

Os profissionais que realizavam o teste de VRG relataram diversidade quanto à fundamentação teórica que subsidia a prática do teste de VRG citando desde a SBNPE até a Comissão Multiprofissional de Alimentação Enteral e Parenteral. Porém, pelo menos um terço dos respondentes relatou desconhecimento quanto à fundamentação teórica que subsidiava sua prática.

O avanço de técnicas e conhecimentos na área da saúde trouxe a necessidade de aperfeiçoamento profissional, vinculado à capacitação continuada e desenvolvimento de habilidades e competências para a excelência do atendimento de enfermagem; para tanto, se faz necessário um maior aprofundamento em conteúdos teóricos e práticos para o melhor cuidado de enfermagem aos pacientes em TNE.

\section{CONCLUSÃO}

Conclui-se que um terço dos enfermeiros desconhece a fundamentação teórica que subsidia sua prática referente ao 
objeto deste estudo, o que pode comprometer a qualidade do atendimento e, por fim, a segurança do paciente.

O teste do VRG foi aplicado, principalmente, para verificar a intolerância alimentar e notou-se que há ausência de consenso em relação aos valores de corte para a prática, padronização da técnica ou padronização de referencial teórico, sendo que para adultos o valor do VRG mais praticado foi de $200 \mathrm{ml}$ e para crianças inferior a $100 \mathrm{ml}$.
Portanto, evidencia-se a necessidade de investigações que colaborem na determinação de quais seriam os protocolos ideais para o cuidado ao paciente, dependendo do tipo de público atendido, favorecendo assim, a redução de falhas na assistência ao suporte nutricional. Além disso, deve-se destacar a necessidade de capacitação profissional adequada para a prática, que envolverá o acompanhamento diário dos procedimentos realizados pela equipe e a educação continuada, colaborando na possível detecção de falhas, qualificando assim a prática assistencial.

\section{RESUMO}

Objetivo: Verificar diferenças na prática de aferição do volume residual gástrico entre enfermeiros clínicos e identificar a fundamentação teórica que subsidia a prática. Método: Estudo transversal realizado por meio do envio de questionário online aos e-mails dos enfermeiros cadastrados no Conselho Regional de Enfermagem do Estado de São Paulo. Resultados: Participam do estudo 598 profissionais de enfermagem, dos quais 484 prestavam assistência apenas a adultos e 114 exclusivamente à crianças. O teste do volume residual gástrico é realizado por $83,4 \%$ dos profissionais da enfermagem, sendo que, em sua maioria, a suspensão e a liberação da terapia nutricional enteral são realizadas pelo médico. A suspensão da terapia nutricional enteral entre adultos ocorre, predominantemente, quando o volume residual gástrico é igual a $200 \mathrm{ml}$ e, entre crianças, se valores menores do que $100 \mathrm{ml}$. A conduta após a suspensão da dieta envolve a devolução do conteúdo gástrico aspirado e a manutenção do cateter fechado até o próximo horário, em 48,3\% dos atendimentos entre adultos e 68,4\% entre crianças. Dos participantes da pesquisa, 42,9\% desconhecem a fundamentação teórica que subsidia a prática do teste. Conclusão: Evidenciou-se a necessidade de capacitação dos enfermeiros e de novas investigações sobre a prática de aferição do volume residual gástrico.

\section{DESCRITORES}

Dieta; Nutrição Enteral; Esvaziamento Gástrico; Cuidados de Enfermagem.

\section{RESUMEN}

Objetivo: Verificar diferencias en la práctica de verificación del volumen gástrico residual entre enfermeros clínicos e identificar la fundamentación teórica que subsidia la práctica. Método: Estudio transversal realizado mediante el envío de cuestionario en línea a los emails de los enfermeros registrados en el Consejo Regional de Enfermería del Estado de São Paulo. Resultados: Participaron en el estudio 598 profesionales enfermeros, de los que 484 prestaban asistencia solo a adultos y 114 exclusivamente a niños. La prueba del volumen gástrico residual la lleva a cabo el 83,4\% de los profesionales enfermeros, siendo que, en su mayoría, la suspensión y la liberación de la terapia de nutrición enteral la realiza el médico. La suspensión de la terapia de nutrición enteral entre adultos ocurre, predominantemente, cuando el volumen gástrico residual es igual a $200 \mathrm{ml}$ y, entre niños, si los valores son menores que $100 \mathrm{ml}$. La conducta después de la suspensión de la dieta involucra la devolución del contenido gástrico aspirado y el mantenimiento del catéter cerrado hasta el próximo horario, en el 48,3\% de las atenciones entre adultos y el 68,4\% entre niños. De los participantes de la investigación, el 42,9\% desconocen la fundamentación teórica que subsidia la práctica de la prueba. Conclusión: Se evidenció la necesidad de capacitación de los enfermeros y de nuevas investigaciones acerca de la práctica de verificación del volumen gástrico residual.

\section{DESCRIPTORES}

Dieta; Nutrición Enteral; Vaciamiento Gástrico; Atención de Enfermería.

\section{REFERÊNCIAS}

1. Martindale RG, Warren M. Should enteral nutrition be started in the first week of critical illness? Curr Opin Clin Nutr Metab Care. 2015;18(2):202-6. DOI: http://dx.doi.org/10.1097/MCO.0000000000000146

2. Elke G, Felbinger TW, Heyland DK. Gastric residual volume in critically ill patients: a dead marker or still alive? Nutr Clin Pract. 2015;30(1):59-71. DOI: http://dx.doi.org/10.1177/0884533614562841

3. Sociedade Brasileira de Nutrição Parenteral e Enteral; Associação Brasileira de Nutrologia. Projeto Diretrizes. Acessos para terapia de nutrição parenteral e enteral [Internet]. São Paulo: AMB/CFM; 2011 [citado 2017 set. 27]. Disponível em: https://diretrizes.amb.org. br/_BibliotecaAntiga/acessos_para_terapia_de_nutricao_parenteral_e_enteral.pdf

4. Taylor BE, McClave SA, Martindale RG, Warren MM, Johnson DR, Braunschweig C, et al. Guidelines for the provision and assessment of nutrition support therapy in the adult critically ill patient: Society of Critical Care Medicine (SCCM) and American Society for Parenteral and Enteral Nutrition (A.S.P.E.N.). Crit Care Med. 2016;44(2):390-438. DOI: http://dx.doi.org/10.1097/CCM.0000000000001525

5. Yip KF, Rai V, Wong KK. Evaluation of delivery of enteral nutrition in mechanically ventilated Malaysian ICU patients. BMC Anesthesiol. 2014;14:127. DOI: https://doi.org/10.1186/1471-2253-14-127

6. Kuppinger DD, Rittler P, Hartl WH, Rüttinger D. Use of gastric residual volume to guide enteral nutrition in critically ill patients: a brief systematic review of clinical studies. Nutrition. 2013;29(9):1075-9. DOI: https://doi.org/10.1016/j.nut.2013.01.025

7. Saran D, Brody RA, Stankorb SM, Parrott SJ, Heyland DK. Gastric vs small bowel feeding in critically ill neurologically injured patients: results of a multicenter observational study. JPEN J Parenter Enteral Nutr. 2015;39(8):910-6. DOI: http://dx.doi.org/10.1177/0148607114540003.

8. Sociedade Brasileira de Nutrição Parenteral e Enteral; Associação Brasileira de Nutrologia. Projeto Diretrizes. Terapia nutricional no paciente grave [Internet]. São Paulo: AMB/CFM; 2011 [citado 2017 set. 27]. Disponível em: https://diretrizes.amb.org.br/_BibliotecaAntiga/ terapia_nutricional_no_paciente_grave.pdf 
9. Cervo AS, Magnago TSBS, Carollo JB, Chagas BP, Oliveira AS, Urbanetto JS. Adverse events related to the use of enteral nutritional therapy. Rev Gaúcha Enferm [Internet]. 2014 [cited 2017 Sep 27];35(2):53-9. Available from: http://www.scielo.br/scielo.php?script=sci_arttext\& pid $=$ S1983-14472014000200053

10. Anziliero F, Côrrea APA, Silva BA, Dal Soller BE, Batassini E, Beghetto MG. Nasoenteral tube: factors associated with delay between indication and use in emergency services. Rev Bras Enferm [Internet]. 2017 [cited 2017 Sep 27];70(2):326-34. Available from: www.scielo. br/scielo.php?script=sci_arttext\&pid=S0034-71672017000200326

11. Zhang Z, Xu X, Ding J, Ni H. Comparison of postpyloric tube feeding and gastric tube feeding in intensive care unit patients: a metaanalysis. Nutr Clin Pract. 2013;28(3):371-80. DOI: http://dx.doi.org/10.1177/0884533613485987

12. Bourgault AM, Heath J, Hooper V, Sole ML, Nesmith EG. Methods used by critical care nurses to verify feeding tube placement in clinical practice. Crit Care Nurse. 2015;35(1):e1-7. DOI: http://dx.doi.org/10.4037/ccn2015984

13. Metheny NA, Mills AC, Stewart BJ. Monitoring for intolerance to gastric tube feedings: a national survey. Am J Crit Care. 2012;21(2):e33-40. DOI: http://dx.doi.org/10.4037/ccn2015984

14. Boullata JI, Carrera AL, Harvey L, Escuro AA, Hudson L, Mays A, et al. ASPEN safe practices for enteral nutrition therapy [Formula: see text]. JPEN J Parenter Enteral Nutr. 2017;41(1):15-103. DOI: http://dx.doi.org/10.1177/0148607116673053

15. Reintam Blaser A, Starkopf L, Deane AM, Poeze M, Starkopf J. Comparison of different definitions of feeding intolerance: a retrospective observational study. Clin Nutr. 2015;34(5):956-61. DOI: https://doi.org/10.1016/j.clnu.2014.10.006

16. Tume L, Carter B, Latten L. A UK and Irish survey of enteral nutrition practices in pediatric intensive care units. Br J Nutr. 2013;109(7):1304-22. DOI: https://doi.org/10.1017/S0007114512003042

17. Tekgündüz KS, Gürol A, Apay SE, Caner I. Effect of abdomen massage for prevention of feeding intolerance in preterm infants. Ital J Pediatr. 2014;40:89. DOI: https://doi.org/10.1186/s13052-014-0089-z

18. Brasil. Ministério da Saúde; Agência Nacional de Vigilância Sanitária. RDC n. 63, de 6 de julho de 2000. Aprova o regulamento técnico para fixar requisitos mínimos exigidos para a terapia de nutrição enteral [Internet]. Brasília: ANVISA; 2000 [citado 2017 set. 27]. Disponível em: https://www20.anvisa.gov.br/segurancadopaciente/index.php/legislacao/item/resolucao-da-diretoria-colegiada-rcd-n-63-de-6-dejulho-de-2000 